\title{
Measurement of the Quadratic Electro-Optic Coefficient of KTN Crystal with an Electro-Optic Modulation System in the Presence of Polar Nano-Regions
}

\author{
Lie-Kun Yang ${ }^{1}$, Bing Liu ${ }^{1, *}$ (D, Pan-Yu Qiao ${ }^{1}$, Hua-Jian Yu ${ }^{1}$, Xu-Ping Wang ${ }^{1}$, Jing Li ${ }^{1,2}$, Yu-Guo Yang ${ }^{1}$, \\ Yuan-Yuan Zhang ${ }^{1}$, Cheng-Cheng Qiu ${ }^{1}$ and Hua-Di Zhang ${ }^{1}$ \\ 1 Advanced Materials Institute, Qilu University of Technology (Shandong Academy of Sciences), \\ Jinan 250014, China; yanglk668@163.com (L.-K.Y.); qpywsry@163.com (P.-Y.Q.); yuhuajian@sdas.org (H.-J.Y.); \\ wangxp@sdas.org (X.-P.W.); lijing2@sdas.org (J.L.); yangyuguo@sdas.org (Y.-G.Y.); \\ zhangyuanyuan@sdas.org (Y.-Y.Z.); qiucc@sdas.org (C.-C.Q.); hdzhang@sdas.org (H.-D.Z.) \\ 2 Shandong Provincial Key Laboratory of High Strength Lightweight Metallic Materials, \\ Advanced Materials Institute, Qilu University of Technology (Shandong Academy of Sciences), \\ Jinan 250014, China \\ * Correspondence: liubing@sdas.org
}

check for updates

Citation: Yang, L.-K.; Liu, B.; Qiao, P.-Y.; Yu, H.-J.; Wang, X.-P.; Li, J.; Yang, Y.-G.; Zhang, Y.-Y.; Qiu, C.-C.; Zhang, H.-D. Measurement of the Quadratic Electro-Optic Coefficient of KTN Crystal with an Electro-Optic Modulation System in the Presence of Polar Nano-Regions. Crystals 2021, 11, 1234. https://doi.org/10.3390/ cryst11101234

Academic Editors: Hassan Hafeez, Sanjoy Paul, Natalie A. Mica and Adel Shaaban Awad

Received: 18 September 2021 Accepted: 11 October 2021 Published: 13 October 2021

Publisher's Note: MDPI stays neutral with regard to jurisdictional claims in published maps and institutional affiliations.

Copyright: () 2021 by the authors. Licensee MDPI, Basel, Switzerland. This article is an open access article distributed under the terms and conditions of the Creative Commons Attribution (CC BY) license (https:// creativecommons.org/licenses/by/ $4.0 /)$.

\begin{abstract}
An electro-optic modulation system was adopted for measuring the quadratic electro-optic coefficient of KTN crystal. Theoretical analysis and experimental results verified the feasibility of this method. The quadratic electro-optic coefficient of a KTN crystal chip, which has a Curie temperature of $0{ }^{\circ} \mathrm{C}$, was measured using this system in the temperature range of $2{ }^{\circ} \mathrm{C}$ to $18{ }^{\circ} \mathrm{C}\left(\mathrm{T}_{\mathrm{C}}=0{ }^{\circ} \mathrm{C}\right)$. The influences of temperature, $\mathrm{AC}$ voltage and frequency on the quadratic electro-optic coefficient were discussed. It was found that the relaxation effect of PNRs (polar nano-regions) played an important role in the determination of the quadratic electro-optic coefficient of KTN crystal.
\end{abstract}

Keywords: KTN single crystal; quadratic electro-optic coefficient; modulation; polar nano-regions

\section{Introduction}

Potassium tantalum niobate has the largest known quadratic electro-optic coefficient in the paraelectric phase [1], which is about 70 times larger than that of lithium niobate [2]. KTN crystal has obvious advantages when it is used as an electro-optic deflector [3] or modulator [4]. The KTN-based optic devices have broad application prospects in the fields of optic communications, biomedical imaging, and radar scanning [5,6]. As early as 2003, high-quality large KTN single crystal with a volume of over $30 \mathrm{~cm}^{3}$ has been grown using the top seeded solution growth method. However, the incongruent melting behavior [7] of KTN crystal growth causes the problem of growth-induced striation, which is generally difficult to overcome. The quadratic electro-optic coefficient of a KTN crystals is usually calculated by measuring its dielectric constant [8], but the obtained result by this method is the average quadratic electro-optic coefficient of the KTN crystal. For the reason of composition inhomogeneity, we cannot accurately measure the quadratic electro-optic coefficient at a certain point by its dielectric constant. By contrast, an optic method is preferred as the more suitable method for the accurate measurement of the quadratic EO coefficient [9]. In this paper, an electro-optic modulation system was adopted for measuring the quadratic electro-optic coefficient of KTN crystal. The influence of temperature, voltage, and frequency on the measurement of the quadratic electro-optic coefficient was obtained.

It is generally believed that the KTN crystal undergoes a phase transition from the paraelectric phase to the ferroelectric phase as the temperature decreases. It is in the paraelectric phase above the Curie temperature, $\mathrm{T}_{\mathrm{c}}$, and in the ferroelectric phase below the Curie temperature. However, recent studies show that the cubic-tetragonal phase transition in KTN is a relaxor-like phase transition [10]. There exists a Burns temperature (TB) above 
the Curie point in the cubic phase of $\mathrm{KTN}$. When $\mathrm{T}_{\mathrm{C}}<\mathrm{T}<\mathrm{TB}$, there are a large number of nano-polar microdomains inside the crystal [11], which makes the paraelectric phase KTN crystal exhibit certain relaxation characteristics. These relaxor-like behaviors also have an impact on the accurate measurement of the quadratic electro-optic coefficient of KTN crystal. This problem will also be discussed in this paper.

\section{Theoretical Analysis}

When a KTN crystal in its cubic phase is placed between a pair of crossed polarizers, the output light intensity will be modulated by the applied electric field on the crystal. Figure 1 is a typical modulation system with a polarizer-sample-analyzer arrangement. The axis of the polarizer is at $45^{\circ}$ of natural birefringence axes of the sample. The output light intensity, I, from the analyzer can be expressed as

$$
\mathrm{I}=\mathrm{I}_{0} \sin \theta^{2} \frac{\Delta \varphi+\varphi_{0}}{2}=\frac{\mathrm{I}_{0}}{2}\left[1-\sin \left(\frac{\pi}{2}-\Delta \varphi-\varphi_{0}\right)\right]
$$

where $\mathrm{I}_{0}$ is the maximum light intensity, and $\varphi_{0}$ is the phase difference caused by natural birefringence [11]. $\Delta \varphi$ is the phase difference caused by the applied voltage under the electro-optic effect, which can be obtained from the following equation [12]:

$$
\Delta \varphi=\frac{2 \pi}{\lambda} \Delta \mathrm{nl}=\frac{\mathrm{n}_{0}^{3}\left(\mathrm{~s}_{11}-\mathrm{s}_{12}\right) \pi \mathrm{l}}{\lambda \mathrm{d}^{2}} \mathrm{~V}^{2}
$$

where $\lambda$ is the laser wavelength, $\Delta \mathrm{n}$ is the refractive index change, $\mathrm{n}_{0}$ is the refractive index of the crystal when no electric field is applied, $\mathrm{d}$ is the distance between the electrodes, 1 is the crystal length in the light direction, $\mathrm{s}_{11}-\mathrm{s}_{12}\left(\mathrm{~s}_{11}, \mathrm{~s}_{12}\right.$, are the abbreviations for the tensor describing the Kerr effect) is the effective quadratic electro-optic coefficient, and V is the applied voltage.

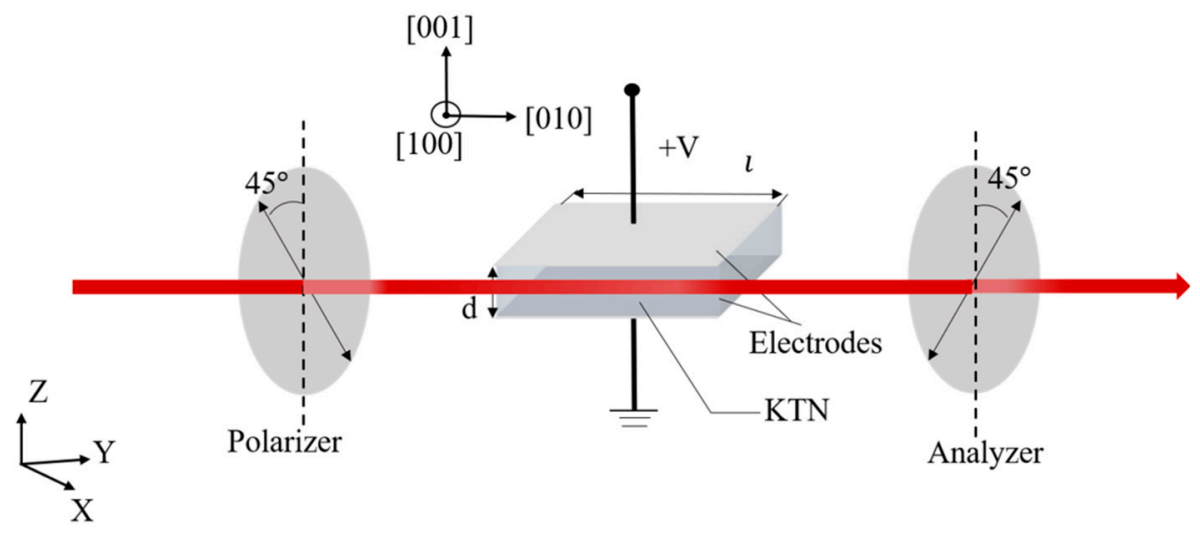

Figure 1. Schematic arrangement of an electro-optic modulation system.

According to Equations (1) and (2), the transmittance curve of a typical KTN modulator is shown in Figure 2. We choose point $\mathrm{M}_{0}^{\prime}$ as the operating point, which is corresponding to the full transmittance at half maximum. The corresponding dc bias voltage at this point is labeled as $\mathrm{V}_{0}$. 


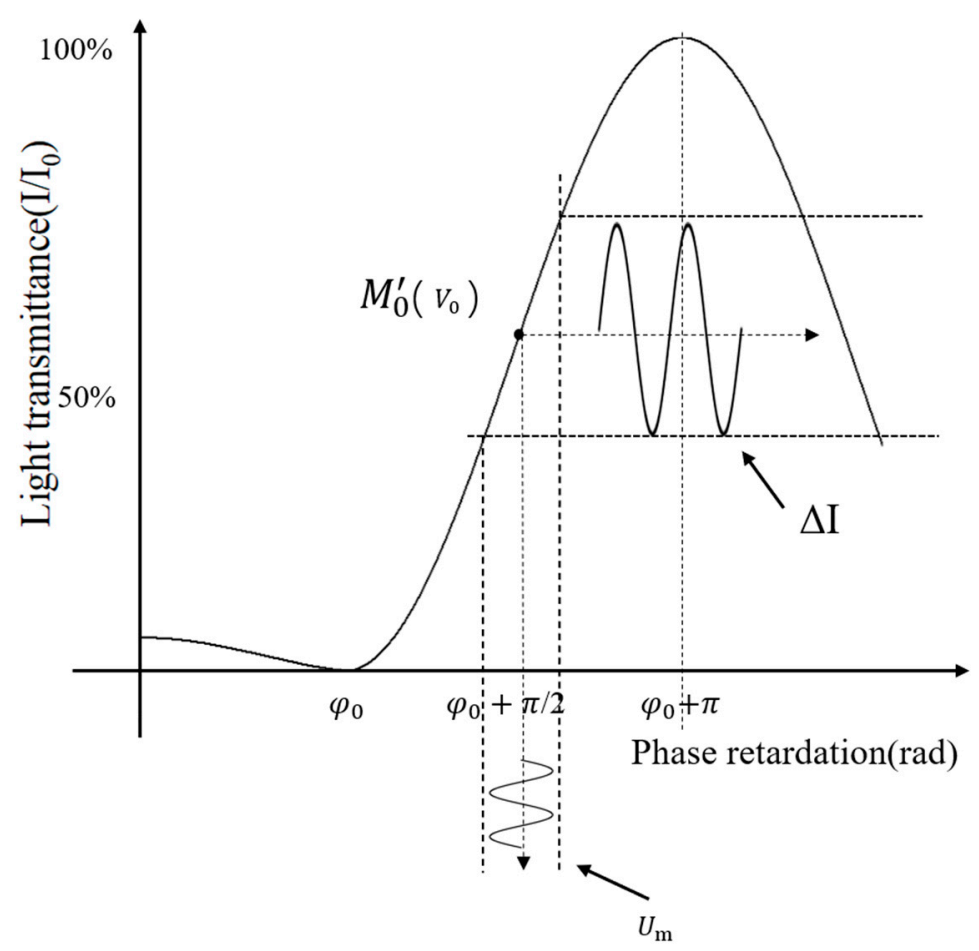

Figure 2. The characteristic transmittance curve of a typical electro-optic modulator.

In Formula (1), when $\frac{\pi}{2}-\Delta \varphi-\varphi_{0}$ approaches zero, that is, the modulator is operated very close to point $\mathrm{M}_{0}^{\prime}$, the small-angle approximation [13] can be used and Equation (1) can be approximately rewritten as

$$
\mathrm{I} \approx \frac{\mathrm{I}_{0}}{2}\left(1-\frac{\pi}{2}+\Delta \varphi+\varphi_{0}\right) .
$$

In practice, the DC bias voltage $\mathrm{V}_{0}$ is used to adjust the work point to $\mathrm{M}_{0}^{\prime}$. Meanwhile, a sinusoidal modulating voltage with an amplitude $U_{m}$ and a frequency $\omega_{m}$ is applied onto the sample at point $\mathrm{M}_{0}^{\prime}$. According to Equations (2) and (3), the output light intensity change $\Delta \mathrm{I}$ caused by the dynamic voltage can be expressed.

$$
\Delta \mathrm{I}=\frac{\mathrm{I}_{0}}{2} \frac{\mathrm{n}_{0}^{3}\left(\mathrm{~s}_{11}-\mathrm{s}_{12}\right) \pi \mathrm{l}}{\lambda \mathrm{d}^{2}}\left(\mathrm{U}_{\mathrm{m}}^{2}+2 \mathrm{~V}_{0} \mathrm{U}_{\mathrm{m}}\right)
$$

where $\mathrm{U}_{\mathrm{m}}$ is the $\mathrm{AC}$ modulation voltage, and $\mathrm{V}_{0}$ is the DC bias voltage. The effective quadratic electro-optic coefficient $\mathrm{s}_{11}-\mathrm{s}_{12}$ can be given by

$$
\mathrm{s}_{11}-\mathrm{s}_{12}=\frac{\lambda \mathrm{d}^{2}}{\pi \ln _{0}^{3}\left(\mathrm{U}_{\mathrm{m}}^{2}+2 \mathrm{~V}_{0} \mathrm{U}_{\mathrm{m}}\right)} \frac{\Delta \mathrm{I}}{\mathrm{I}_{0} / 2}
$$

where the maximum light intensity $\mathrm{I}_{0}$, the dc bias voltage $\mathrm{V}_{0}$ at the half-maximum transmittance point, and output light intensity change $\Delta \mathrm{I}$ caused by the dynamic voltage can be measured in the experiment. Hence, the effective quadratic electro-optic coefficient $\mathrm{s}_{11}-\mathrm{s}_{12}$ can be obtained using the electro-optic modulation system in Figure 1.

\section{Results and Discussion}

The high-quality $\mathrm{KTa}_{0.65} \mathrm{Nb}_{0.35} \mathrm{O}_{3}$ single crystal was grown from the mixed solution of $\mathrm{KTaO}_{3}(\mathrm{KT})$ and $\mathrm{KNbO}_{3}(\mathrm{KN})$ with a top-seeded solution growth method. We confine the three lengths of the block of $4 \mathrm{~mm}, 6 \mathrm{~mm}$, and $2.5 \mathrm{~mm}$ to the three crystal axes $\mathrm{x}, \mathrm{y}$, and $\mathrm{z}$, respectively. Two parallel light transmission surfaces were polished. Another two parallel surfaces normal to the direction of the applied electric field were sputtered with 
platinum electrode. The relative dielectric constant of the KTN crystal chip as a function of temperature could be obtained by a LCR digital bridge (HIOKI 3532-50) which is shown in Figure 3. Through the dielectric thermograph data, we get that the Curie temperature of this KTN crystal chip is $0^{\circ} \mathrm{C}$.

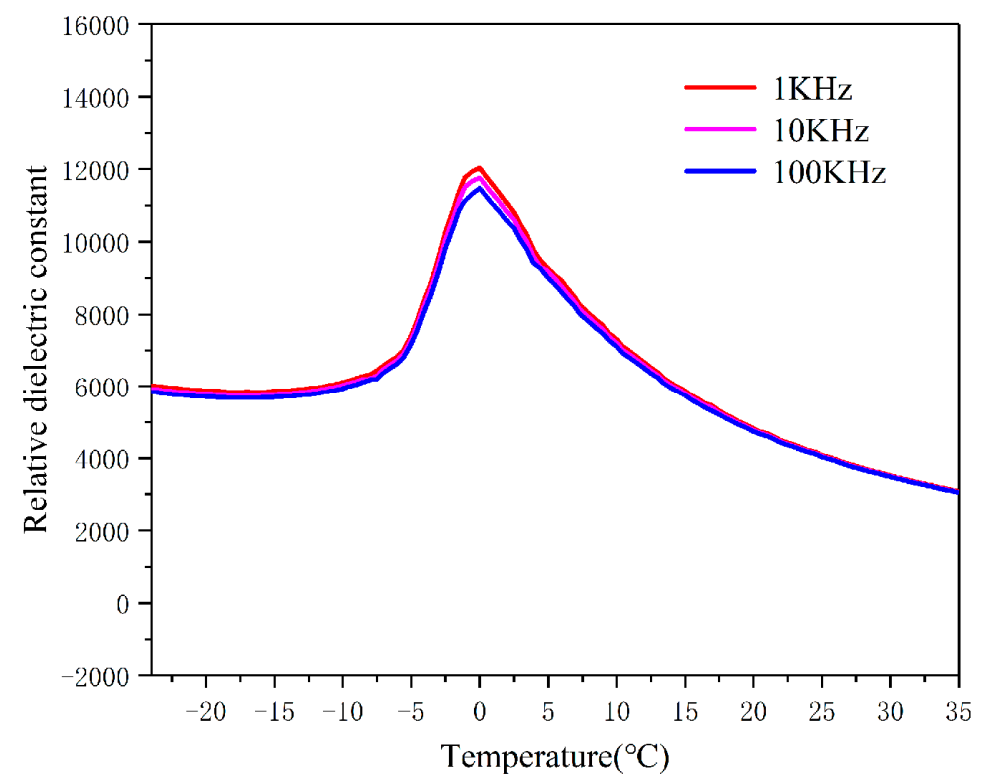

Figure 3. Relative permittivity.

The experiment arrangement of the electro-optic modulation system for the quadratic electro-optic coefficient of KTN crystal is shown in Figure 4. The temperature of the KTN crystal chip was controlled by a TEC temperature controller with an accuracy of $\pm 0.1^{\circ} \mathrm{C}$. In order to ensure the temperature control accuracy and avoid water condensation on the crystal surface, the KTN sample and the TEC module were placed in a vacuum box. The light source is a linearly polarized He-Ne laser with $\lambda=632.8 \mathrm{~nm}$. KTN crystal is placed between the crossed polarizer $(\mathrm{P})$ and analyzer $(\mathrm{A})$. The polarizing direction of the polarizer is set at $45^{\circ}$ to the optic axis of the crystal. The voltage signal generated by the signal generator (RIGOL DG4062) is applied to the crystal through a high voltage amplifier (PINTECH HA-800). When the laser beam passes through the iris diaphragm and the ND filter, the spot diameter and the beam intensity are both reduced. Then, the beam is modulated by the polarizer-sample-analyzer system and the output light is converted into a voltage signal by the photodetector.

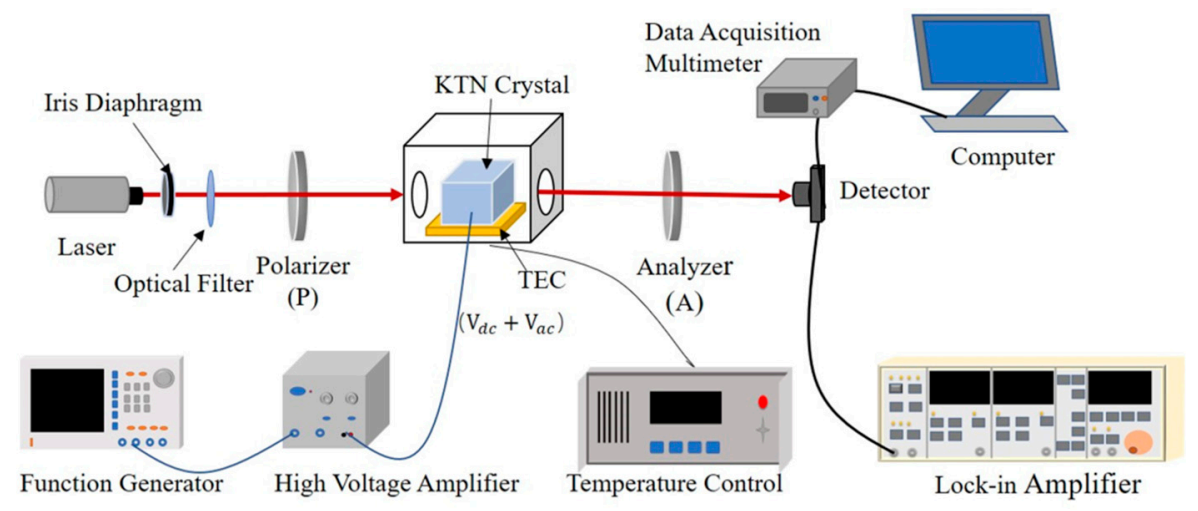

Figure 4. Schematic diagram of the experimental light path.

When a variable DC voltage is applied to the crystal, the output voltage signal from the detector, as well as the voltage applied to the crystal, was recorded by the data acquisition 
multimeter (Keithley DAQ6510). A typical light transmittance curve is shown in Figure 5. We can determine the voltage $\mathrm{V}_{0}$ applied on the crystal at the operating point $\mathrm{M}_{0}^{\prime}$. We can also determine the maximum voltage output $\mathrm{v}_{(\mathrm{p}-\mathrm{p})}$ from the detector, which is proportional to the maximum output light intensity. When $A C$ voltage with amplitude $U_{m}$ is applied at the operating point $\mathrm{M}_{0}^{\prime}$, the amplitude of the output voltage signal $\mathrm{v}_{\text {out }}$ can be measured by a lock-in amplifier (Stanford SR830), and what we need to pay attention to is that the receiving frequency of the lock-in amplifier is $2 \omega_{\mathrm{m}}$. Finally, we get the equation

$$
\frac{\Delta \mathrm{I}}{\mathrm{I}_{0} / 2}=\frac{\mathrm{v}_{\text {out }}}{\mathrm{v}_{(\mathrm{p}-\mathrm{p})} / 2}
$$

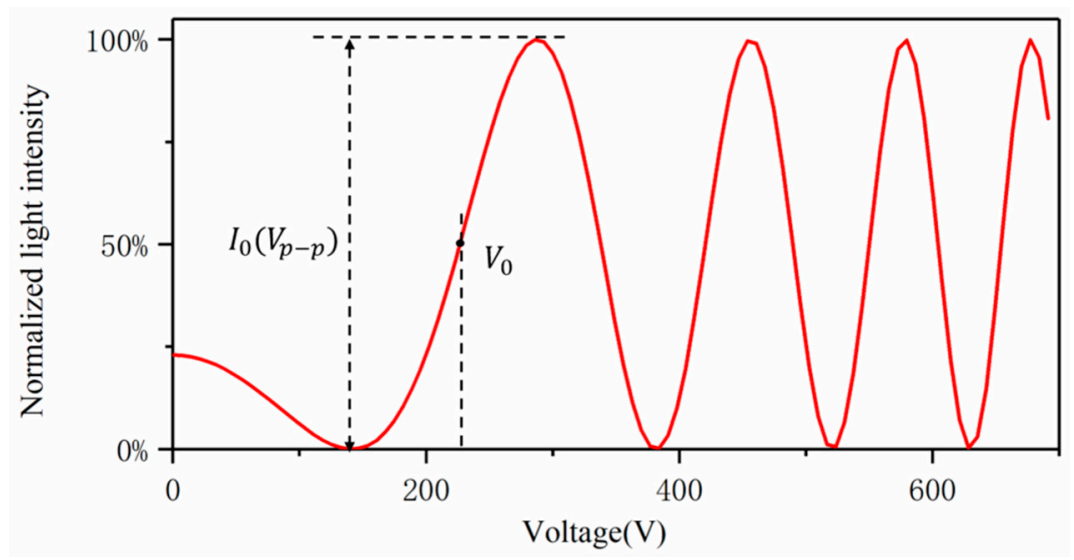

Figure 5. Light intensity modulation curve when a sinusoidal voltage of 0 to $700 \mathrm{~V}$ is applied at $15^{\circ} \mathrm{C}$.

From Equations (6) and (7), the quadratic electro-optic coefficient can be calculated using the following formula:

$$
\mathrm{s}_{11}-\mathrm{s}_{12}=\frac{\lambda \mathrm{d}^{2}}{\pi \ln _{0}^{3}\left(\mathrm{U}_{\mathrm{m}}^{2}+2 \mathrm{~V}_{0} \mathrm{U}_{\mathrm{m}}\right)} \frac{\mathrm{v}_{\text {out }}}{\mathrm{v}_{(\mathrm{p}-\mathrm{p})} / 2} .
$$

Figure 6 shows the quadratic electro-optic coefficients of points A, B, and C at different temperatures. In the temperature range of $2-18{ }^{\circ} \mathrm{C}$, the quadratic electro-optic coefficients of the three points all show a similar trend, and the quadratic electro-optic coefficients all increase rapidly as the temperature gets closer to the phase transition point. As mentioned earlier, in the TB interval above the Curie temperature, there are polar nano-domains in the crystal [11]. So, as the temperature decreases, the size of the polar nano-region becomes larger and larger orderliness is also enhanced. Hence the electric susceptibility increases and the electro-optic effect is correspondingly enhanced. When the crystal is above the Curie temperature, its relative permittivity can be described by the Curie-Weiss law [14]:

$$
\frac{1}{\varepsilon_{\mathrm{r}}}-\frac{1}{\varepsilon_{\mathrm{m}}}=\frac{\left(\mathrm{T}-\mathrm{T}_{\mathrm{m}}\right)^{\gamma}}{\mathrm{C} \prime}
$$

where $\varepsilon_{\mathrm{r}}$ is the relative permittivity, $\varepsilon_{\mathrm{m}}$ represents the maximum value of the relative permittivity, $\mathrm{T}_{\mathrm{m}}$ represents the maximum characteristic temperature corresponding to the maximum value of the permittivity, which is approximately equal to the Curie temperature, $\mathrm{T}_{\mathrm{C}}, \mathrm{C}^{\prime}$ is the corrected Curie-Weiss constant, and $\gamma$ is the relaxation factor that quantifies the degree of dispersion of ferroelectric materials. 

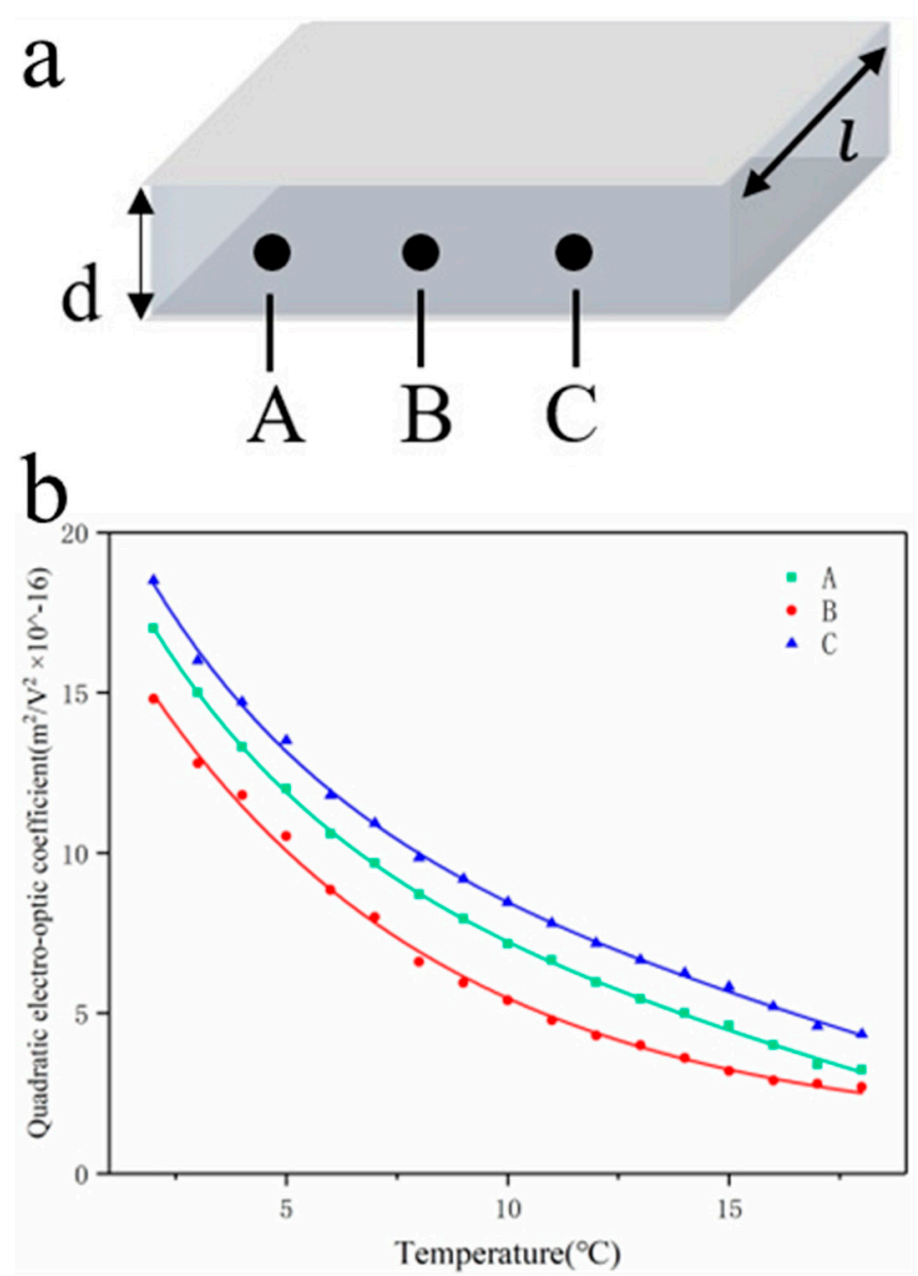

Figure 6. (a) The position of A, B, and C on the crystal. (b) The quadratic electro-optic coefficients at three different positions of $\mathrm{A}, \mathrm{B}$, and $\mathrm{C}$ in the range of $2-18^{\circ} \mathrm{C}$ (the solid line is the fitting result).

The relationship between the relative permittivity $\varepsilon$ and the effective quadratic electrooptic coefficient is [15]

$$
\mathrm{s}_{11}-\mathrm{s}_{12}=\left(\mathrm{g}_{11}-\mathrm{g}_{12}\right) \varepsilon_{0}^{2}\left(\varepsilon_{\mathrm{r}}-1\right)^{2} \approx\left(\mathrm{g}_{11}-\mathrm{g}_{12}\right) \varepsilon_{0}^{2} \varepsilon_{\mathrm{r}}^{2}
$$

where $g_{11}$ and $g_{12}$ are the quantifying nonlinearity of the medium, the change in the refractive index with the optical intensity. According to reports, the coefficients of KTN are $\mathrm{g}_{11}=0.136 \mathrm{~m}^{4} / \mathrm{C}^{2}$ and $\mathrm{g}_{12}=-0.038 \mathrm{~m}^{4} / \mathrm{C}^{2}$ [16]. Therefore, we can know that the relative permittivity $\varepsilon_{\mathrm{r}}$ of the crystal will continue to increase as the temperature decreases, and the quadratic electro-optic coefficient will also increase.

In Figure 6, we also noticed that the quadratic electro-optic coefficients at different positions of a certain crystal are significantly different. This is due to the fact that the incongruent melting behavior of KTN crystal growth causes the growth-induced striation, which makes the Curie temperature at these three points slightly different, so the electrical susceptibility and dielectric constant of each point at the same temperature are different. Being able to accurately measure the quadratic electro-optic coefficients at different points is also one of the advantages of this system.

As mentioned earlier, the DC and AC superimposition method was used in the experiment. DC is used to control the operating point to $\mathrm{I}_{0} / 2$, and the amplitude of the AC voltage is variable under the condition of meeting the small-angle approximation. As shown in Figure 7, we record the quadratic electro-optic coefficients under different AC voltages. We found that the quadratic electro-optic coefficient of the crystal will decrease 
rapidly and they tend to be stable with the increase of modulation voltage. The influence of AC voltage on $s_{11}-s_{12}$ is more significant when the temperature is close to the crystal Phase transition point. We believe that the increased AC electric field suppresses the reorientation of PNRs which contributes the electro-optic effect. With the increment of the modulation voltage, the inhibition effect is saturated and the electro-optic effect tends to be stable.

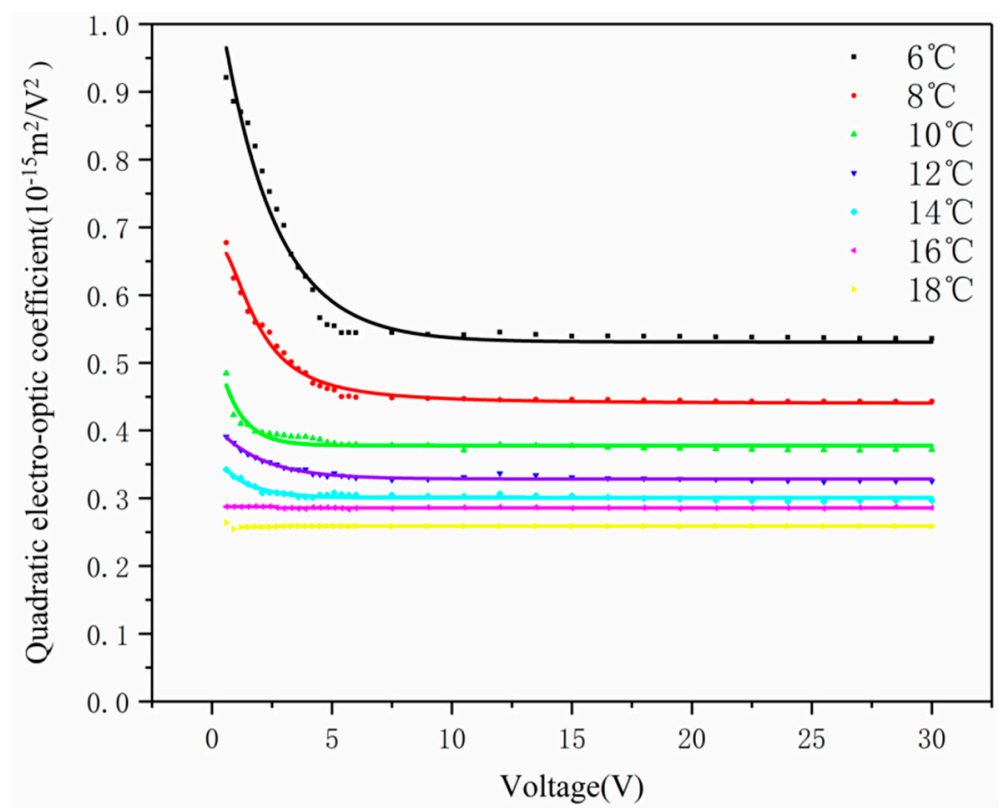

Figure 7. The quadratic electro-optical coefficients under different AC modulation voltages in the temperature range of $6-18{ }^{\circ} \mathrm{C}$ (the solid line is the fitting result).

We did not measure complete data in the range from $2{ }^{\circ} \mathrm{C}$ to $5{ }^{\circ} \mathrm{C}$ degrees Celsius, since we found that there is a linear electro-optical effect in this range. For a relaxor KTN crystal near the Curie temperature, the change of refractive index originates from intrinsic contribution (polarizable lattice) and extrinsic contribution (reorientation and vibration of PNRs). Therefore, the macroscopic polarization $P$ is composed of $\mathrm{p}_{\mathrm{PNR}}$ contributed by PNR and linear susceptibility $\chi_{p}$ due to the paraelectric host, which can be expressed as [17]

$$
\mathrm{P}=\mathrm{p}_{\mathrm{PNR}}+\mathrm{p}_{\mathrm{X}_{\mathrm{p}}}=\rho \mathrm{p}_{0} \tanh \left[\frac{\mathrm{p}_{0}|\mathrm{E}|}{\mathrm{k}_{\mathrm{B}}\left(\mathrm{T}-\mathrm{T}_{0}\right)}\right] \mathrm{u}+\varepsilon_{0} \mathrm{X}_{\mathrm{p}} \mathrm{E} .
$$

Following the quadratic electro-optic effect, the resulting refractive change is

$$
\begin{gathered}
\Delta \mathrm{n}=-\left(\frac{1}{2}\right) \mathrm{n}^{3}\left(\mathrm{~g}_{11}-\mathrm{g}_{12}\right) \varepsilon_{0}^{2} \chi_{\mathrm{p}}^{2} \mathrm{E}^{2}-\mathrm{n}^{3}\left(\mathrm{~g}_{11}-\mathrm{g}_{12}\right) \rho \mathrm{p}_{0} \tanh \left[\frac{\mathrm{p}_{0}|\mathrm{E}|}{\mathrm{k}_{\mathrm{B}}\left(\mathrm{T}-\mathrm{T}_{0}\right)}\right] \varepsilon_{0} \chi_{\mathrm{p}}|\mathrm{E}| \\
-\left(\frac{1}{2}\right) \mathrm{n}^{3}\left(\mathrm{~g}_{11}-\mathrm{g}_{12}\right) \rho^{2} \mathrm{p}_{0}^{2} \tanh ^{2}\left[\frac{\mathrm{p}_{0}|\mathrm{E}|}{\mathrm{k}_{\mathrm{B}}\left(\mathrm{T}-\mathrm{T}_{0}\right)}\right]
\end{gathered}
$$

where $T_{0}$ is the freezing temperature, $u$ is the field unit vector, $\rho$ is the density of clusters with dipole moment $\mathrm{p}_{0}, \mathrm{k}_{\mathrm{B}}$ is the Boltzmann's constant the first term indicates the quadratic dependence on the electric field that can be neglected due to the small $\chi_{p}$ contributed by the by paraelectric host, the second term of the above formula implies the linear relationship between the refractive index change and the electric field when $\tan \mathrm{h}\left[\mathrm{p}_{0}|\mathrm{E}| / \mathrm{k}_{\mathrm{B}}\left(\mathrm{T}-\mathrm{T}_{0}\right)\right]=1$, and the third term suggests the quadratic dependence under the low electric field. Therefore, the increased electric field can induce the linear electro-optic effect.

As we know, there is a double frequency relationship between the applied AC voltage and the modulated light intensity when a quadratic electro-optic effect plays a role, so we can judge whether it is a linear electro-optic effect or a quadratic electro-optic effect by oscilloscope traces of the signal of modulation voltage and detector response. Under the 
low electric field, the optical signal indicates that the quadratic effect plays a leading role. With the increment of the electric field, linear and quadratic effects coexist. The electric field further increases, the linear effect is dominated.

The frequency of the AC voltage applied on the crystal chip is also a variable in the measurement procedure. As shown in Figure 8, we have recorded the dependence of quadratic electro-optic coefficient with modulation frequency in the temperature range of $2{ }^{\circ} \mathrm{C}<\mathrm{T}-\mathrm{T}_{\mathrm{c}}<18{ }^{\circ} \mathrm{C}$ with the increase of electric field frequency, the quadratic electrooptic coefficient gradually decreases and finally tends to a stable value. This is due to the inevitable composition inhomogeneity of the KTN crystals, which makes it impossible for the size of the PNRs to be completely consistent. In an AC electric field, the dielectric permittivity will increase, which is attributed to the PNRs' reorientation activated [18]. The quadratic electro-optic effect is stronger at a low frequency. However, with the increase of the frequency of the electric field, the large-size PNRs can no longer be reoriented with the electric field. Therefore, its contribution to the quadratic electro-optic effect remains unchanged, resulting in the decrease of the quadratic electro-optic coefficient. This is more pronounced near the Curie temperature. In addition, PNRs are polar at the nanoscale and can behave as nanoresonators [19]. When the crystal is close to the Curie temperature, a larger size PNR is often formed. The quadratic electro-optic effect is very strong, which is due to the stronger resonance phenomenon at low frequencies. However, as the frequency of the electric field increases, the resonance phenomenon weakens, and the quadratic electro-optic coefficient attenuates. The PNRs become smaller in size when the KTN crystals are far from the Curie temperature. Therefore, the resonance phenomenon is very weak at each frequency, and the quadratic electro-optic coefficient no longer attenuates even if the frequency increases.

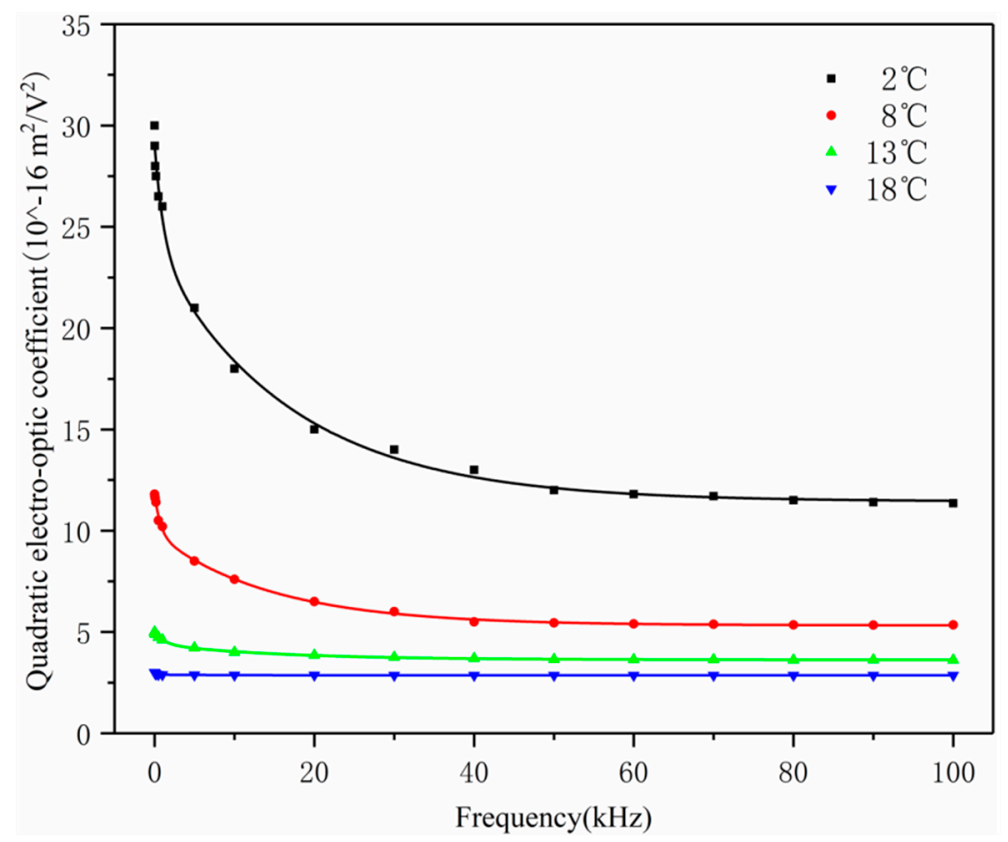

Figure 8. The quadratic electro-optic coefficients of AC modulation voltage frequency in the range of $10-100 \mathrm{kHz}$ (the solid line is the fitting result).

Through the previous experiments, it can be known that temperature, AC modulation voltage, and frequency have a great influence on the quadratic electro-optic coefficient, and it is more significant when it is close to the Curie temperature. In the previous experiment, we did not know whether the position of the operating point has changed, so we decided to test the stability of the operating point position. As shown in Figure 9, we recorded multiple complete transmittance curve waveforms at $\mathrm{T}=5^{\circ} \mathrm{C}$. We can see that the curves basically coincide after two or three voltage cycles, which indicates that the PNRs in the 
crystal reached an equilibrium state. When the inside of the crystal is polarized, the PNR also reaches an equilibrium state. The polarization characteristics in KTN crystals mainly come from the displacement of niobium ions [20]. The microscopic local symmetry of KTN crystals is broken when voltage is applied to the crystal, and then the dipoles on the lattice scale appear. As the applied voltage increases, the random movement of polarization of dipoles in the crystal decreases, and they may group to form larger-sized PNRs [20,21]. This causes the refractive index of different areas inside the crystal to become different [22]. Random scattering occurs when incident light enters, resulting in crystal depolarization and attenuation of the modulation depth. This polarization gradually saturates as the voltage continues to be applied. The modulation curve no longer changes, since the state of PNRs becomes stable. This also shows that the position of the operating point has not changed during the experiment.

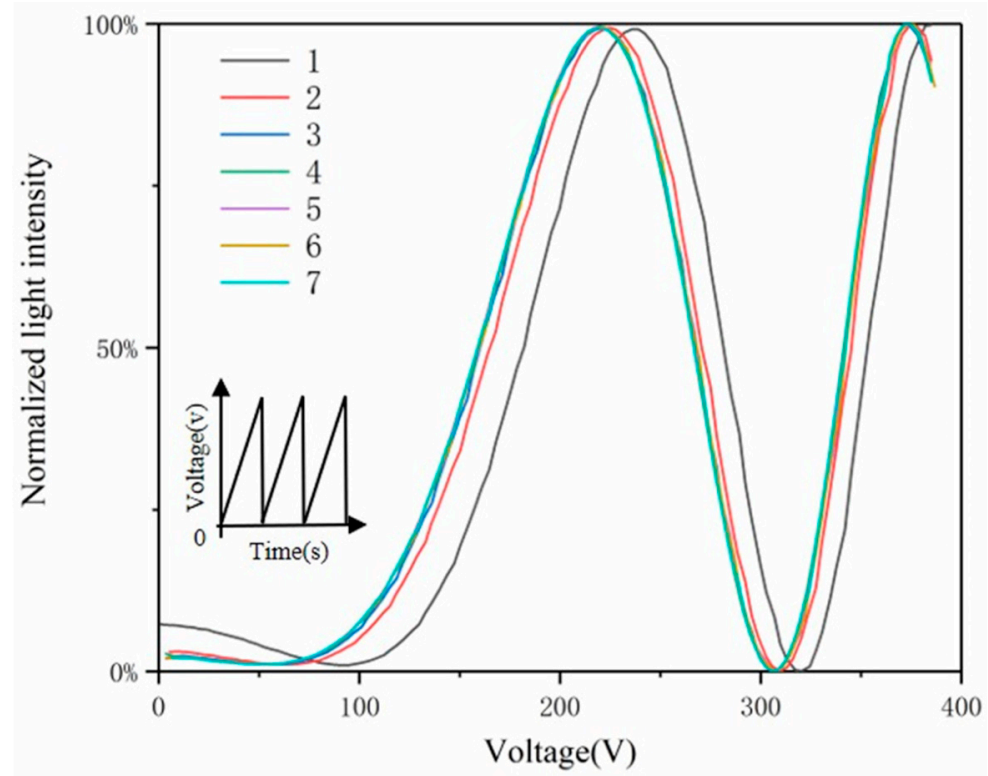

Figure 9. Modulated transmittance curves with a sawtooth wave voltage. (The inset of the lower left corner of the figure is the applied sawtooth wave voltage, and the numbers represent each cycle of the applied voltage.).

The above problems will be encountered in practical applications, and we also need to notice them. The study of these influencing factors allows us to further understand the change law of the quadratic electro-optic coefficient of KTN crystal, which helps us to grow more homogeneity crystals and more accurately characterize the quadratic electro-optic coefficient of KTN crystals.

\section{Conclusions}

In this study, a quadratic electro-optic coefficient measurement system was introduced, and the effective quadratic electro-optic coefficient calculation formula is obtained through theoretical derivation. This optic method can more accurately measure the quadratic electrooptic coefficient of a certain position of the crystal. The effects of temperature, voltage, and frequency on the quadratic electro-optic coefficient were analyzed, and the relaxation effect of PNRS was discussed. The quadratic electro-optic coefficient increases significantly when it is close to the Curie temperature, and this is attributed to the effect of temperature on polar nano-regions. The incongruent melting behavior of KTN crystal growth causes growthinduced striation, which makes the quadratic electro-optic coefficients of different positions have obvious differences. When the temperature drops close to the phase transition point, the high modulation voltage significantly attenuates the quadratic electro-optic coefficient. Furthermore, the frequency response of PNRs of different sizes is different. Generally, the quadratic electro-optic coefficient is higher at low frequencies than at high 
frequencies. PNRs under the action of an electric field have a response time, and the position of the operating point will not change after reaching a stable state. A more accurate characterization of the quadratic electro-optic coefficient of KTN crystal is helpful to the practical application of KTN crystal devices. However, how to avoid these problems encountered in stability requires further exploration.

Author Contributions: L.-K.Y. (writing-original draft preparation), B.L. (writing-review and editing), P.-Y.Q. (resources), H.-J.Y. (formal analysis), X.-P.W. (project administration), J.L. (visualization), Y.-G.Y. (investigation), Y.-Y.Z. (methodology), C.-C.Q. (funding acquisition), and H.-D.Z. (software). All authors have read and agreed to the published version of the manuscript.

Funding: This research was funded by National Natural Science Foundation of China, grant numbers 51972179, 51902168 and 52072189; Natural Science Foundation of Shandong Province, grant numbers ZR2020KE019 and ZR2020QE041); Science and Technology Program for Young Innovation Team in Colleges and Universities of Shandong Province, China, grant number 2019KJA003; Studio for the Leader of Scientific Research in Jinan, grant number 2019GXRC059 and the Project for Introduced Innovation Team in Jinan, grant numbers 2020GXRC037.

Conflicts of Interest: The authors declare no conflict of interest.

\section{References}

1. Fujiura, K.; Nakamura, K. KTN optical waveguide devices with an extremely large electro-optic effect. Proc. SPIE 2005, 5623, 518-532.

2. Wang, C.; Zhang, M.; Stern, B.; Lipson, M.; Loncar, M. Nanophotonic lithium niobate electro-optic modulators. Opt. Express 2018, 26, 1547-1555. [CrossRef] [PubMed]

3. Tian, F.; Lu, H.; Zhan, S.; Tao, J.; Zhe, C. Electro-optic deflection in a lithium niobate quasi-single mode waveguide with microstructured electrodes. Opt. Express 2018, 26, 30100-30107. [CrossRef] [PubMed]

4. Chang, Y.C.; Wang, C.; Yin, S.; Hoffman, R.C.; Mott, A.G. Kovacs effect enhanced broadband large field of view electro-optic modulators in nanodisordered KTN crystals. Opt. Express 2013, 21, 17760-17768. [CrossRef] [PubMed]

5. Miyazu, J.; Imai, T.; Toyoda, S.; Sasaura, M.; Yagi, S.; Kato, K.; Sasaki, Y.; Fujiura, K. New beam scanning model for high-speed operation using $\mathrm{KTa}_{1-\mathrm{x}} \mathrm{Nb}_{\mathrm{x}} \mathrm{O}_{3}$ crystals. Appl. Phys. Express 2011, 4, 111501. [CrossRef]

6. Xu, J.; Zhi, Y.; Wang, X.; Sun, J.; Liu, L. A large-angle high speed scanner based on electro-optic crystal for Fresnel telescope synthetic aperture imaging ladar. Proc. SPIE 2012, 8520, 85200V.

7. Imai, T.; Sasaura, M.; Nakamura, K.; Fujiura, K. Crystal growth and electro-optic properties of $\mathrm{KTa}_{1-\mathrm{x}} \mathrm{Nb}_{\mathrm{x}} \mathrm{O}_{3}$. NTT Tech. Rev. 2007, $5,1-8$.

8. Yang, C.-K.; Wang, X.-P.; Zhang, F.; Zhang, H.-D.; Liu, B.; Li, J.; Zhang, Y.-Y.; Yang, Y.-G.; Lv, X.-S.; Wei, L. Electro-optic intensity modulation in Fe-doped $\mathrm{KTa}_{0.65} \mathrm{Nb}_{0.35} \mathrm{O}_{3}$ crystals. Crystals 2020, 10, 870. [CrossRef]

9. Aillerie, M.; Theofanous, N.; Fontana, M.D. Measurement of the electro-optic coefficients: Description and comparison of the experimental techniques. Appl. Phys. B 2000, 70, 317-334. [CrossRef]

10. Rahaman, M.M.; Imai, T.; Miyazu, J.; Kobayashi, J.; Kojima, S. Relaxor-like dynamics of ferroelectric $\mathrm{K}\left(\mathrm{Ta}_{1-\mathrm{x}} \mathrm{Nb} \mathrm{b}_{\mathrm{x}}\right) \mathrm{O} 3 \mathrm{crystals}$ probed by inelastic light scattering. J. Appl. Phys. 2014, 116, 074110. [CrossRef]

11. Ziebinska, A.; Rytz, D.; Szot, K.; Gorny, M.; Roleder, K. Birefringence above $\mathrm{T}_{\mathrm{c}}$ in single crystals of barium titanate. J. Phys.-Condens. Mat. 2008, 20, 142202. [CrossRef]

12. Gupta, S.; Huband, S.; Keeble, D.S.; Walker, D.; Thomas, P.; Viehland, D.; Priya, S. Optical crystallographic study of piezoelectric $\mathrm{K}_{\mathrm{x}} \mathrm{Na}_{1-\mathrm{x}} \mathrm{NbO}_{3}(\mathrm{x}=0.4,0.5$ and 0.6) single crystals using linear birefringence. CrystEngComm 2013, 15, 6790-6799. [CrossRef]

13. Aillerie, M.; Fontana, M.; Abdi, F.; Carabatos-Nedelec, C.; Theofanous, N. Accurate measurement of the electro-optic coefficients: Application to $\mathrm{LiNbO}_{3}$. Proc. SPIE 1989, 1018, 94-101.

14. Bokov, A.A.; Ye, Z.G. Dielectric relaxation in relaxor ferroelectrics. J. Adv. Dielectr. 2012, 2, 1241010. [CrossRef]

15. Geusic, J.E.; Kurtz, S.K.; Uitert, L.V.; Wemple, S.H. Electro-optic properties of some $\mathrm{ABO}_{3}$ perovskites in the paraelectric phase. Appl. Phys. Lett. 1964, 4, 141-143. [CrossRef]

16. Lu, Q.; Han, J.; Dai, H.; Ge, B.; Zhao, S. Temperature dependence of Kerr coefficient and quadratic polarized optical coefficient of a paraelectric Mn:Fe:KTN crystal. AIP Adv. 2015, 5, 087117. [CrossRef]

17. Viehland, D.; Li, J.F.; Jang, S.J.; Cross, L.E.; Wuttig, M. Dipolar-Glass Model for Lead Magnesium Niobate. Phys. Rev. B 1991, 43 , 8316-8320. [CrossRef] [PubMed]

18. Tan, P.; Tian, H.; Mao, C.; Hu, C.; Meng, X.; Li, L.; Shi, G.; Zhou, Z. Field-driven electro-optic dynamics of polar nanoregions in nanodisordered $\mathrm{KTa}_{1-\mathrm{x}} \mathrm{Nb}_{\mathrm{x}} \mathrm{O}_{3}$ crystal. Appl. Phys. Lett. 2017, 111, 012903. [CrossRef]

19. Cai, L.; Pattnaik, R.; Lundeen, J.; Toulouse, J. Piezoelectric polar nanoregions and relaxation-coupled resonances in relaxor ferroelectrics. Phys. Rev. B 2018, 98, 134113. [CrossRef] 
20. Delre, E.; Spinozzi, E.; Agranat, A.J.; Conti, C. Scale-free optics and diffractionless waves in nano-disordered ferroelectrics. Nat. Photonics 2011, 5, 39-42. [CrossRef]

21. Parravicini, J.; Agranat, A.J.; Conti, C.; Delre, E. Equalizing disordered ferroelectrics for diffraction cancellation. Appl. Phys. Lett. 2012, 101, 911-912. [CrossRef]

22. Xin, Z.; Shan, H.; Zhao, Z.; Wu, P.; Liu, H. Abnormal optical anisotropy in correlated disorder $\mathrm{KTa}_{1-\mathrm{x}} \mathrm{Nb}_{\mathrm{x}} \mathrm{O}_{3}: \mathrm{Cu}_{\text {with refractive }}$ index gradient. Sci. Rep. 2018, 8, 2892. 